\title{
ВОЗМОЖНЫЕ Я: ОБЗОР ЗАРУБЕЖНЫХ ИССЛЕДОВАНИЙ
}

\author{
Е.Ю. ВАСИЛЕВСКАЯ
}

${ }^{a}$ Национальный исследовательский университет «Высшая школа экономики», 101000, Россия, Москва, ул. Мясницкая, д. 20

\begin{abstract}
Резюме
В статье представлен обзор зарубежных исследований возможных Я. Возможные Я понимаются как представления индивида о том, каким он хотел бы стать, мог бы стать или боится стать в будущем. Раскрываются свойства возможных Я. Возможные Я рассматриваются как часть Я-концепции, представленной когнитивными репрезентациями индивидуально значимых целей, стремлений, надежд и опасений, в которых происходит связь настоящего и будущего личности. Отмечается важность текущего социокультурного и исторического контекста для формирования возможных Я, а также их уязвимость и изменчивость по сравнению с другими элементами Я-концепции. Выделяются основные виды возможных Я: ожидания, надежды и опасения, а также лучшие возможные Я и потерянные возможные Я. Проводится детальный анализ параметров возможных Я, среди которых названы: эмоциональный знак, сбалансированность, наличие/отсутствие стратегии достижения возможного Я, детальность, воспринимаемая эффективность, субъективная значимость, частота обращения. Рассматривается регулятивная функция возможных Я, заключающаяся в стимуляции и организации будущего поведения, придании дополнительного смысла и значения текущему поведению, в оценке жизненного опыта и происходящих изменений в жизни личности, в управлении эмоциональными процессами. Раскрывается понятие мотивационного потенциала возможных Я, в котором происходит пересечение мотивации и Яконцепции. Анализируются результаты исследований, направленных на поиск и выявление параметров возможных Я, которые обладают наибольшим мотивационным потенциалом. Описываются методы изучения возможных Я, важнейшими из которых являются опросники, интервью и эксперименты. В заключении обозначаются нерешенные проблемы в области изучения возможных Я и перспективы дальнейших исследований.
\end{abstract}

Ключевые слова: возможные Я, Я-концепция, мотивационный потенциал.

В последнее время в гуманитарных дисциплинах, в том числе в пси- хологии, все бо́льшую популярность приобретают исследования категорий 
«возможного» и «возможностей» (Абульханова-Славская, 1991; Петровский, 2015; Старовойтенко, 2013; Эпштейн, 2001). Как указывает В.А. Петровский, «Я - это сгусток возможностей, воплощаемых вовне, а главная потребность человеческого бытия - освоение этих возможностей» (Петровский, 2010, с. 368). Наиболее полно идея «возможного» в отечественной психологии отражена в концепции личностного потенциала Д.А. Леонтьева, которая позволяет обозначить контуры и теоретические основания нового подхода в персонологии (Леонтьев, 2011). Феномен возможных Я занимает особое место в области исследования категории «возможного» в психологии. Однако если в зарубежных психологических журналах ему посвящены сотни исследований, то в отечественных - лишь единицы.

Впервые данный феномен получил подробное содержательное раскрытие в публикации X. Маркус и П. Нуриус в 1986 г., которые определяют возможные Я как представления человека о том, каким он хотел бы стать, мог бы стать или боится стать в будущем (Markus, Nurius, 1986). Возможные Я - это когнитивные репрезентации целей, стремлений, мотивов, надежд, опасений и страхов индивида, которые являются частью его Я-концепции.

В отличие от идеального Я, традиционно считавшегося одним из важнейших образований Я (Роджерс, 1997; Фрейд, 2015; Хорни, 1997; и др.), возможные Я включают в себя не только позитивные элементы, но и негативные - страхи, опасения и т.д.; возможные Я тяготеют к конкретным и индивидуализированным представлениям о себе в будущем, тогда как идеальные Я являются более абстрактными элементами Яконцепции, заданными социальными нормативами (Белинская, 1999), приближаясь скорее к жизненным задачам, типичным у людей определенной возрастной категории (Erikson, 2007); возможные Я обладают достаточно мощным мотивационным потенциалом, требующим их реализации, в то время как идеальные Я нередко бывают формальными, не выполняющими регулирующей функции. Возможные Я как представленные в настоящий момент образы будущего наиболее полно отражены в метафоре «когнитивного моста» между планами «настоящего Я» и «будущего Я» или «дорожной карты» (Oyserman et al., 2004; и др.). В этом конструкте отражается то, что Блаженный Августин называл «настоящее будущего» (Блаженный Августин, 1998). С точки зрения Д. Ойзерман и Х. Маркус, возможные Я придают особую форму, смысл и направление динамичным элементам личности (Oyserman, Markus, 1990). На наш взгляд, возможные Я позволяют не только удерживать временну́ю направленность Я, но и учитывать вариативность этих будущих Я, следовательно, осознанный их выбор как индивидуально приемлемых жизненных ориентиров, а также ответственность личности за этот выбор.

\section{Свойства, виды, параметры и функции возможных Я}

Х. Маркус и П. Нуриус выделяют следующие свойства возможных «Я» (Markus, Nurius, 1986). Возможные 
«Я» - совокупность индивидуально значимых надежд, страхов и фантазий человека, а не любых воображаемых ролей или представлений о будущем. Именно переживание их значимости для своего Я позволяет индивиду сравнивать реальные и желаемые представления о себе, создавая между ними напряжение и направляя поведение в сторону его уменьшения (Markus, Ruvolo, 2015). Представления о будущем, которые не связаны с Я индивида, безразличны ему, не обладают столь сильной мотивацией, в строгом смысле их нельзя считать возможными Я (Erikson, 2007).

Возможные $Я$ формируются в текущем социокультурном и историческом контексте. Возможные Я находятся в тесной связи с окружающей социальной средой, формируются в отношениях со значимыми другими (Cameron, 1999; Oyserman et al., 1995; Unemori et al., 2004; и др.). Среда представляет индивиду образцы, модели жизни, которые он примеряет на себя и которые могут стать прицельными точками в будущем, т.е. возможными Я.

Возможные Я являются наиболее уязвимыми и изменчивыми представлениями человека о себе. В отличие от более или менее устойчивых реальных Я возможные Я, будучи фантазиями, целями, мечтами, могут преобразовываться, исчезать, возникать вновь при изменении внешних и внутренних условий.

В исследованиях возможных Я (Carver et al., 1994; King, Hicks, 2007; King, Patterson, 2001; Markus, Nurius, 1986; и др.) упоминаются различные их вариации, среди которых можно выделить следующие виды: 1) ожи- дания («Я, которыми я могу стать») возможные Я, которыми индивид хочет и может стать в будущем; 2) надежды ( «Я, которыми я надеюсь cтать») - возможные Я, которыми индивид хочет, но не может легко стать в будущем; 3) опасения («Я, которыми я боюсь стать») - возможные Я, которыми индивид не хочет, но может стать в будущем; 4) лучшие возможные Я (best possible selves) - наиболее значимые возможные Я, связанные с достижением важных для индивида целей и задающие направление жизни человека на достаточно длительные промежутки времени; 5) потерянные возможные Я (lost possible selves) - представления о себе в будущем, которые раньше существовали в опыте индивида, но сейчас уже не являются частью его жизни.

Кроме того, в литературе можно встретить выделение различных параметров возможных Я: эмоциональный знак (представления о себе в будущем могут быть как позитивными, так и негативными или эмоционально нейтральными). Полагают, что ожидания и надежды преимущественно, но не обязательно являются позитивными, а опасения - негативными возможными Я; сбалансированность (сбалансированные возможные Я - это такие возможные Я, у которых ожидания и опасения касаются одной и той же области, но они противоположны по содержанию. Например, ожидание - получить высшее образование, опасение «завалить» сессию и быть отчисленным из вуза); наличие/отсутствие стратегии достижения возможного $Я$ означает степень представления индивида о том, как воплотить в 
жизнь его возможное Я, какие шаги необходимо для этого предпринять; детальность, отражающая степень проработанности возможного Я; воспринимаемая әффективность, обозначающая степень возможности воплотить в жизнь (в случае надежды или ожидания) или избежать воплощения (в случае опасения) тех или иных возможных Я; субъективная значимость, связанная с тем, насколько возможные Я затрагивают личностные ценности и смыслы; частота обращения отражает количество времени, уделяемого размышлениям о том или ином возможном Я и ряд других параметров (Hooker, 1992; King, Smith, 2004; Oyserman et al., 2004; Oyserman, Markus, 1990).

Введение большого количества все новых и новых параметров обусловлено тем, что исследователи пытаются определить, какие именно возможные Я обладают мотивационным потенциалом и воплощаются в реальности.

Поскольку возможные Я многоплановы, нередко их различают по сферам жизни человека. Наиболее исследованными являются возможные Я, связанные с учебной (академическая успеваемость, карьерные планы), межличностной (супружеские и детско-родительские отношения) и внутриличностной (идентичность, психологическое благополучие, оптимизм, здоровье, отношение к своему телу) сферами (Bybee, Wells, 2006; Carver et al., 1994; Dunkel, 2000; Dunkel, Anthis, 2001; Hooker, 1992; Morfei et al., 2001; Oyserman et al., 2006; Ryff, 1991; и др.).

В зависимости от направления, в котором ведется исследование возможных Я и круга проблем, привле- кающих внимание автора, описываются различные функции возможных Я. Наиболее существенной, с точки зрения разных исследователей, является регулятивная функция: возможные Я рассматриваются как стимул и организатор будущего поведения; возможные Я придают дополнительный смысл и значение текущему поведению индивида, а также оценке его жизненного опыта и происходящих изменений (Erikson, 2007; Freeman et al., 2001; Markus, Nurius, 1986; Ryff, 1991; и др.); возможные Я регулируют эмоциональные процессы, повышая оптимизм и уверенность в том, что изменение возможно (Oyserman et al., 2004).

\section{Мотивационный потенциал и динамика возможных Я}

Одним из самых популярных направлений изучения возможных Я является исследование различий в их содержании в разных возрастных группах или в разных жизненных обстоятельствах (Hooker, 1992; Ryff, 1991). Проводятся исследования возможных Я у подростков с различной степенью делинквентности; у пожилых людей в сравнении с более молодыми; у мужчин до и после рождения ребенка; у лиц, преодолевших личностный кризис; у молодых взрослых во время поиска работы, у представителей различных культур, у клиентов в процессе психотерапии и др. (Dunkel et al., 2006; Hooker, Kaus, 1994; Markus, Nurius, 1986; Morfei et al., 2001; Oyserman, Markus, 1990; и др.). Показано, например, что в репертуаре возможных Я молодых взрослых представления о себе как 
будущем родителе в наименьшей степени были выражены у респондентов из Японии по сравнению с канадцами и итальянцами (Bloom et al., 1999). В других исследованиях выявлено, что в подростковом возрасте возможные Я чаще связаны с учебой, межличностными отношениями, личностными качествами, материальными объектами и др. (Oyserman, Markus, 1990), в поздней юности превалируют возможные Я в области личностных качеств, стиля жизни, внешности, профессии, навыков и способностей (Markus, Nurius, 1986), в поздней взрослости - возможные Я, связанные со здоровьем, семьей, независимостью и стилем жизни (Frazier et al., 2000).

В исследованиях, выполненных в данном русле, констатируются лишь различия в возможных Я или их динамика; вопрос о том, как они влияют на поведение человека, зачастую не поднимается.

Особое направление в исследовании возможных Я представляют работы, связывающие данный феномен с мотивацией. Если традиция изучения мотивации в психологии практически полностью исключает роль Я-концепции в поведении человека (Markus, Ruvolo, 2015), то в области исследования возможных Я связь Я-концепции и мотивации приобретает ключевое значение. В самом общем виде можно сказать, что возможные Я обладают мотивационным потенциалом, они, будучи образами себя в будущем, способны организовывать поведение человека в направлении желаемого состояния (Oyserman, Markus, 1990; и др.).

Уже в первых экспериментальных исследованиях мотивационного по- тенциала возможных Я было показано, что представление позитивных возможных Я улучшает эффективность выполнения действий, в то время как представление негативных возможных Я снижает еe (Markus, Nurius, 1986). Однако в ряде других исследований было зафиксировано, что негативные возможные Я обладают бо́льшим мотивационным потенциалом, чем позитивные. К примеру, негативные образы себя в будущем в области здоровья оказались больше связаны с текущим поведением, направленным на его поддержание (Hooker, Kaus, 1994).

По мнению некоторых исследователей, позитивность или негативность возможных Я вовсе не является определяющим параметром повышения мотивации (Oyserman et al., 2015; и др.). В настоящее время существует немалое количество работ, направленных на поиск и выявление параметров возможных Я, которые обладают наибольшим мотивационным потенциалом. Среди них называют сбалансированность, разработанность, соотношение контекста и эмоционального знака возможных Я, метафоры возможных Я, наличие стратегии их достижения и т.д. Выявлено, к примеру, что чем более разработанным и сбалансированным является возможное Я, тем больше вероятность того, что оно станет направлять поведение человека (Markus, Ruvolo, 2015). Имеются данные о том, что индивид воплощает в жизнь не весь репертуар своих возможных Я, а лишь те из них, которые содержат стратегии достижения желаемых образов себя в будущем, и этот параметр более значим, чем сбалансированность 
возможных Я (Oyserman et al., 2004; и др.). Доказывается, что возможные Я, сопряженные со стратегиями их достижения, являются предикторами академической успеваемости, хорошего поведения в школе, а также уменьшения депрессивных симптомов. Для некоторых групп респондентов, например детей, воспитывающихся бабушками и дедушками, существование большого количества образов будущего без стратегий достижения целей чревато нарушением поведения (Bi, Oyserman, 2015).

Осознание того, что возможное Я в принципе может быть воплощено в жизнь конкретным субъектом, также повышает его мотивационный потенциал (Oyserman et al., 2006). В этом случае возможное Я переживается как конгруэнтное идентичности индивида. Например, ученики, полагающие, что их профессиональный успех в будущем зависит от текущей академической успеваемости, уделяли больше времени учебе в отличие от учащихся, которые так не думали (Destin, Oyserman, 2010).

Определенную роль в воплощении в жизнь возможных Я играет социальный контекст, поддерживающий или, наоборот, препятствующий этому процессу (Kao, 2000; Oyserman et al., 2006; и др.). Д. Ойзерман с соавт. показали, что стереотипные представления, связанные с тем, что афроамериканские студенты меньше способны к высоким академическим достижениям, приводят к тому, что эти студенты испытывают сложности в реализации своих возможных Я в академической сфере (Oyserman et al., 2002). Осознание принадлежности к группе большинства и удовлетворенность членством в группе, напротив, вызывает уверенность в том, что надежды удастся воплотить в жизнь, а опасения предотвратить (Cameron, 1999).

Еще одним параметром, влияющим на повышение мотивации, являются метафорические описания возможных Я в виде путешествия (или дороги) к желаемому будущему, которые создают более четкое представление о связи текущего и желаемого состояния, а также этапов достижения этого предпочитаемого будущего (Landau et al., 2014). В исследованиях показано, что респонденты, которым предъявлялись данные метафоры, проявляли бо́льшую заинтересованность в мероприятиях и материалах, направленных на помощь в подготовке к экзаменам, больше усилий тратили на выполнение заданий и достигали лучшего результата в отличие от участников, которые представляли свои возможные Я, используя метафору контейнера или вовсе без метафор. Важно отметить, что данный эффект проявлялся лишь в ситуации, когда респонденты воспринимали это метафорическое путешествие как проявление собственных усилий.

Можно выделить еще одну тенденцию в изучении мотивационного потенциала возможных Я - это кросскультурные исследования. В качестве примера отметим исследование, в котором было установлено, что сбалансированность возможных Я повышает мотивационный потенциал достижения желаемых образов будущего у европейцев и американцев, в то время как у японцев, напротив, именно совпадение возможных Я (например, ожидание - стать безработным, опасение - стать безработным) 
усиливает мотивацию достижения своих целей (Unemori et al., 2004).

C самого начала исследования возможных Я были направлены на решение не только теоретических, но и прикладных задач: разрабатывались программы обучения, серии тренинговых занятий, повышающих мотивационный потенциал возможных Я путем проработки стратегий их достижения, установления позитивных связей между возможными Я и значимыми социальными идентичностями и т.д. (Hock et al., 2006; Oyserman et al., 2002, 2006).

\section{Методы изучения возможных Я}

Для исследования возможных Я применяется разнообразный репертуар методов - от опросников и интервью до экспериментов, артметодов, описаний отдельных терапевтических случаев и нарративных описаний.

Наиболее часто используемыми являются опросные методики, которые существуют в самых разных вариантах: опросник Х. Маркус и П. Нуриус, позволяющий получить широкий репертуар возможных Я конкретного индивида (Markus, Nurius, 1986); опросник изучения Яконцепции Д. Ойзерман и Х. Маркус с открытыми и закрытыми вопросами, направленными на оценку того, насколько приведенная возможность характеризует человека в настоящее время, насколько она может стать его характеристикой в будущем и в какой степени он хотел бы видеть ее в будущем (Oyserman, Markus, 1990); опросник К. Хукер, помимо фиксации возможных Я, позволяет изучить некоторые их характеристики - воспринимаемую эффективность, ожидаемый результат, частоту мыслей, значимость (Hooker, 1992); опросник изучения возможных Я в следующем году Д. Ойзерман с соавт., направленный на исследование не только самих возможных Я, но и стратегий их достижения (Oyserman et al., 2004). Применяемые для исследования возможных Я интервью зачастую являются устной формой опросников (Hooker, 1992).

Примером арт-методов может служить работа с рисунком дерева возможных Я, когда участник исследования изображает дерево, ветвями, листьями, корнями и другими элементами которого являются образы себя в будущем, а затем начинает с ним работать, выстраивая конкретные цели, связанные с его возможными Я, и продумывая пути их достижения (Hock et al., 2006).

В последнее время популярность приобретают нарративные методы исследования возможных Я - нарративное интервью или открытые вопросы анкеты, подразумевающие продуцирование респондентом историй о себе в будущем, которые еще не были прожиты и не проживаются в реальности, но могут возникать в сознании индивида как возможный вариант жизни (King, Raspin, 2004; и др.). Отмечается, что нарративный метод может быть более полезным по сравнению с опросниками, так как не ограничивает респондентов в фантазировании (Whitty, 2002).

Наибольший интерес вызывают экспериментальные исследования возможных Я, направленные на изучение их мотивационного влияния, регулятивной функции. Подобные исследования можно разделить 
на две большие группы. К первой группе относятся лабораторные эксперименты (например: Cross, Markus, 1994), в которых вначале испытуемые продуцируют возможные Я, а затем им предъявляют задачу (например, умножение и деление трехзначных чисел в уме и т.д.), успешность выполнения которой можно оценить с помощью объективных показателей. Таким образом удается изучить влияние возможных Я и их параметров на успешность решения задачи (Landau et al., 2014; Markus, Nurius, 1987).

Ко второй группе относятся исследования преимущественно в области педагогической психологии, проводимые в естественных условиях. Типичное исследование строится следующим образом. На первом этапе измеряются возможные Я респондентов, а также их успеваемость, отношение к школе, активность на уроках и т.д. Далее через определенное время (обычно через год) делается повторный замер этих параметров. С помощью таких исследований удалось, например, выявить положительный вклад возможных Я, связанных со стратегиями их достижения, в активность учащихся на уроках, в их успеваемость, поведение в школе и снижение депрессивных симптомов (Bi, Oyserman, 2015; Oyserman et al., 2004).

В серии других исследований было показано, что проведение специальных тренинговых занятий со школьниками и студентами, направленных на формирование образов себя в будущем, позволяет им, с одной стороны, продуцировать больше возможных Я, связанных с академической сферой, с другой, претво- рять их в свою реальную жизнь. По сравнению с контрольными группами участники тренинга более активно проявляли себя на занятиях, больше времени тратили на выполнение домашних заданий, имели более высокую успеваемость, большее их число успешно окончили университет (Hock et al., 2006; Oyserman et al., 2002, 2006).

Важно отметить, что основной массив данных по изучению возможных Я получен с помощью опросных методов, которые не позволяют ответить на ряд вопросов, в частности, оценить влияние возможных Я на поведение человека. Только расширение экспериментальных исследований возможных Я, выход их из исследований исключительно академической сферы позволит наметить пути решения вопросов, связанных с мотивационным потенциалом возможных Я.

\section{Заключение}

Проведенный обзор показал нарастающую тенденцию в эмпирическом изучении возможных Я, все больше внимания уделяется мотивационной силе образов желательного и нежелательного Я в будущем. Возможные Я позволяют заполнить лакуну между формальным идеалом и реальным Я, пролить свет на механизмы осуществления самодетерминации, прояснить факторы, мотивирующие выбор человеком тех, а не иных целей.

В то же время обзор зарубежных исследований позволил выявить ряд проблем, связанных с конструктом возможных Я. В первую очередь, это проблемы более точного очерчивания 
той реальности, которая стоит за понятием «возможные Я»; определения устойчивых конфигураций параметров возможных Я, которые могут выступить в качестве предикторов целеобразования; изучение мотивационного потенциала не только надежд и опасений, но и других, выделяемых исследователями типов возможных Я - потерянных, оставленных, фантазийных и т.д.; оценка роли возможных Я в защите самоуважения и в межличностных отношениях (нередко мы выбираем «свой круг» в зависимости от общих устремлений, целей и намерений); анализ возможных Я как механизма смыслообразования, самоопределения и самопроектирования.

Представляется, что концептуальное осмысление феномена возможных Я, который вобрал в себя не только значение структурного компонента Я-концепции, но и временну́ю направленность личности, интенцию к ее динамике и развитию, необходимо вести под углом зрения двух направлений - Я-концепции и временно́й перспективы. В настоящее время в фокусе исследований находится первое направление и неоправданно мало исследований, которые пытались бы отрефлексиро- вать и осмыслить данный феномен с позиции вопросов целеполагания, уровня притязаний и временно́й трансспективы. Несмотря на большое количество исследований, посвященных мотивационному потенциалу возможных Я, в целом создается довольно мозаичная картина, преобладают частные результаты без попытки их теоретического осмысления, выделения общих механизмов и закономерностей перехода возможных Я в реальные цели человека, рассмотрения движущих сил этого перехода и построения основных этапов превращения идеальных Я в возможные Я, а последних - в реализованные.

Исследование возможных Я, направленное на прояснение взаимодействия возможных и реальных Я, позволит подойти к решению проблемы влияния представлений человека о собственном будущем на его поведение в ситуации «здесь-итеперь», на выбор и трансформацию жизненных целей, на инициирование целенаправленного действия, на принятие решений, на конструирование смысла. Можно сказать, что изучение сферы возможного - это во многом будущее психологии и других наук.

\section{Литература}

Абульханова-Славская, К. А. (1991). Стратегия жизни. М.: Мысль.

Белинская, Е. П. (1999). Временные аспекты Я-концепции и идентичности. Мир психологии, 3 , $140-147$.

Блаженный Августин. (1998). Творения (т. 1). СПб./Киев: Алетейя/УЦИММ-Пресс.

Леонтьев, Д. А. (ред.). (2011). Личностный потенциал: структура и диагностика. М.: Смысл.

Петровский, В. А. (2010). Человек над ситуацией. М.: Смысл.

Петровский, В. А. (2015). Субъектность как состоятельность. Психология. Журнал Высшей школь экономики, 12(3), 86-130. (на англ. яз.) 
Роджерс, К. (1997). Клиентощентрированная психотерапия. М./Киев: Рефл-бук/Ваклер.

Старовойтенко, Е. Б. (2013). Возможности Я в отношении к другому: герменевтика и рефлексия. Психология. Журнал Высшей школы экономики,10(4), 121-142.

Фрейд, 3. (2015). Я и Оно. СПб.: Азбука.

Хорни, К. (1997). Невроз и личностный рост. Борьба за самоосуществление. СПб.: ВосточноЕвропейский институт психоанализа и БСК.

Эпштейн, М. Н. (2001). Философия возможного. СПб.: Алетейя.

Bi, C., \& Oyserman, D. (2015). Left behind or moving forward? Effects of possible selves and strategies to attain them among rural Chinese children. Journal of Adolescence, 44, 245-258. doi:10.1016/ j.adolescence.2015.08.004

Bloom, K., Delmore-Ko, P., Masataka, N., \& Carli, L. (1999). Possible self as parent in Canadian, Italian, and Japanese young adults. Canadian Journal of Behavioural Science, 31(3), 198-207. doi: $10.1037 / \mathrm{h} 0087088$

Bybee, J. A., \& Wells, Y. V. (2006). Body themes in descriptions of possible selves: Diverse perspectives across the life span. Journal of Adult Development, 13(2), 95-101. doi:10.1007/s10804-006-9009-9

Cameron, J. E. (1999). Social identity and the pursuit of possible selves: Implications for the psychological well-being of university students. Group Dynamics: Theory, Research, and Practice, 3(3), 179-189. doi:10.1037/1089-2699.3.3.179

Carver, C., Reynolds, S., \& Scheier, M. (1994). The possible selves of optimists and pessimists. Journal of Research in Personality, 28(2), 133-141. doi:10.1006/jrpe.1994.1011

Cross, S. E., \& Markus, H. R. (1994). Self-schemas, possible selves, and competent performance. Journal of Educational Psychology, 86(3), 423-438. doi:10.1037/0022-0663.86.3.423

Destin, M., \& Oyserman, D. (2010). Incentivizing education: Seeing schoolwork as an investment, not a chore. Journal of Experimental Social Psychology, 46(5), 846-849. doi:10.1016/j.jesp.2010.04.004

Dunkel, C. S. (2000). Possible selves as a mechanism for identity exploration. Journal of Adolescence, 23(5), 519-529. doi:10.1006/jado.2000.0340

Dunkel, C. S., \& Anthis, K. S. (2001). The role of possible selves in identity formation: a short-term longitudinal study. Journal of Adolescence, 24(6), 765-776. doi:10.1006/jado.2001.0433

Dunkel, C. S., Kelts, D., \& Coon, B. (2006). Possible selves as mechanisms of change in therapy. In C. Dunkel, J. \& Kerpelman (Eds.), Possible selves: Theory, research, and application (pp. 187-204). Huntington, NY: Nova.

Erikson, M. (2007). The meaning of the future: Toward a more specific definition of possible selves. Review of General Psychology, 11(4), 348-358. doi:10.1037/1089-2680.11.4.348

Frazier, L. D., Hooker, K., Johnson, P. M., \& Kaus, C. R. (2000). Continuity and change in possible selves in later life: A 5-year longitudinal study. Basic and Applied Social Psychology, 22(3), 237243. doi:10.1207/S15324834BASP2203_10

Freeman, M., Hennessy, E., \& Marzullo, D. (2001). Defensive evaluation of antismoking messages among college-age smokers: The role of possible selves. Health Psychology, 20(6), 424-433. doi:10.1037//0278-6133.20.6.424

Hock, M. F., Deshler, D. D., \& Schumaker, J. B. (2006). Enhancing student motivation through the pursuit of possible selves. In C. Dunkel \& J. Kerpelman (Eds.), Possible selves: Theory, research, and application (pp. 205-221). Huntington, NY: Nova.

Hooker, K. (1992). Possible selves and perceived health in older adults and college students. Journal of Gerontology, 47(2), 85-95. doi:10.1093/geronj/47.2.P85 
Hooker, K., \& Kaus, C. R. (1994). Health-related possible selves in young and middle adulthood. Psychology and Aging, 9(1), 126-133. doi:10.1037/0882-7974.9.1.126

Kao, G. (2000). Group images and possible selves among adolescents: Linking stereotypes to expectations by race and ethnicity. Sociological Forum, 15(3), 407-430. doi:10.1023/A:1007572209544

King, L. A., \& Hicks, J. A. (2007). Whatever happened to "What might have been?" Regrets, happiness, and maturity. American Psychologist, 62(7), 625-636. doi:10.1037/0003-066X.62.7.625

King, L. A., \& Patterson, C. (2001). Reconstructing life goals after the birth of a child with Down Syndrome: Finding happiness and growing. International Journal of Rehabilitation and Health, 5(1), 14-21. doi:10.1023/A:1012955018489

King, L. A., \& Raspin, C. (2004). Lost and found possible selves, subjective well-being, and Ego development in divorced women. Journal of Personality, 72(3), 603-632. doi:10.1111/j.00223506.2004.00274.x

King, L. A., \& Smith, N. G. (2004). Gay and straight possible selves: Goals, identity, subjective wellbeing, and personality development. Journal of Personality, 72(5), 967-994. doi:10.1111/j.00223506.2004.00287.x

Landau, M. J., Keefer, L. A., Oyserman, D., \& Smith, G. C. (2014). The college journey and academic engagement: How metaphor use enhances identity-based motivation. Journal of Personality and Social Psychology, 106(5), 679-698. doi:10.1037/a0036414

Markus, H., \& Nurius, P. (1986). Possible selves. American Psychologist, 41(9), 954-969. doi: 10.1037/0003-066X.41.9.954

Markus, H., \& Nurius, P. (1987). Possible selves: The interface between motivation and the self-concept. In K. Yardley \& T. Honess (Eds.), Self and identity: Psychosocial perspectives (pp. 157-172). Oxford: Wiley \& Sons.

Markus, H., \& Ruvolo, A. (2015). Possible selves: Personalized representations of goals. In L. A. Pervin (Ed.), Goal concepts in personality and social psychology (pp. 211-241). London/New York: Psychology Press.

Morfei, M. Z., Hooker, K., Fiese, B. H., \& Cordeiro, A. M. (2001). Continuity and change in parenting possible selves: A longitudinal follow-up. Basic and Applied Social Psychology, 23(3), 217-223. doi:10.1207/153248301750433777

Oyserman, D., Bybee, D., \& Terry, K. (2006). Possible selves and academic outcomes: How and when possible selves impel action. Journal of Personality and Social Psychology, 91(1), 188-204. doi:10.1037/0022-3514.91.1.188

Oyserman, D., Bybee, D., Terry, K., \& Hart-Jonson, T. (2004). Possible selves as roadmaps. Journal of Research in Personality, 38(2), 130-149. doi:10.1016/S0092-6566(03)00057-6.

Oyserman, D., Destin, M., \& Novin, S. (2015). The context-sensitive future self: Possible selves motivate in context, not otherwise. Self and Identity, 14(2), 173-188. doi:10.1080/ 15298868.2014.965733

Oyserman, D., Gant, L., \& Ager, J. (1995). A socially contextualized model of African American identity: Possible selves and school persistence. Journal of Personality and Social Psychology, 69(6), 1216-1232. doi:10.1037/0022-3514.69.6.1216

Oyserman, D., \& Markus, H. (1990). Possible selves and delinquency. Journal of Personality and Social Psychology, 59(1), 112-125. doi:10.1037/0022-3514.59.1.112

Oyserman, D., Terry, K., \& Bybee, D. (2002). A possible selves intervention to enhance school involvement. Journal of Adolescence, 25(3), 313-326. doi:10.1006/jado.2002.0474 
Ryff, C. D. (1991). Possible selves in adulthood and old age: A tale of shifting horizons. Psychology and Aging, 6(2), 286-295. doi:10.1037/0882-7974.6.2.286

Unemori, P., Omoregie, H., \& Markus, H. (2004). Self-portraits: Possible selves in EuropeanAmerican, Chilean, Japanese and Japanese-American cultural contexts. Self and Identity, 3(4), 321-338. doi:10.1080/13576500444000100

Whitty, M. (2002). Possible selves: Exploring the utility of a narrative approach. Identity: An International Journal of Theory and Research, 2(3), 213-230. doi:10.1207/S1532706XID0203_02

Василевская Екатерина Юрьевна - аспирант, факультет социальных наук, департамент психологии, кафедра общей и экспериментальной психологии, Национальный исследовательский университет «Высшая школа экономики».

Сфера научных интересов: Я-концепция, возможные Я, мотивация, психология личности. Контакты: evasilevskaya@hse.ru

Молчанова Ольга Николаевна - профессор, факультет социальных наук, департамент психологии, кафедра общей и экспериментальной психологии, Национальный исследовательский университет «Высшая школа экономики», кандидат психологических наук, доцент.

Сфера научных интересов: Я-концепция, самооценка, психология развития.

Контакты: omolchanova@hse.ru

\title{
Possible Selves: Review of International Studies
}

\author{
E.Yu. Vasilevskaya ${ }^{a}$, O.N. Molchanova ${ }^{a}$ \\ ${ }^{a}$ National Research University Higher School of Economics, 20 Myasnitskaya str., Moscow, 101000, \\ Russian Federation
}

\begin{abstract}
The article presents a review of foreign studies on possible selves. Possible selves are understood as individual's notions on what one would like to become or is afraid to become in the future. Properties of possible selves are described. Possible selves are viewed as part of self-concept that manifests in cognitive representations of individually significant goals, aspirations, hopes and fears, which provide continuity between the present and the future of personality. The relevance of the current social cultural and historical context for the development of possible selves is discussed, as well as their vulnerability and variability in comparison to other elements of self-concept. Main types of possible selves are identified: expectations, hopes and fears, as well as best possible selves and lost possible selves. A detailed analysis of the parameters of possible selves, among which are emotional valence, balance, presence or absence of a strategy to reach possible self, articulation, perceived effectiveness, subjective significance, frequency of reference. The regulative function of possible selves is discussed, which consists in stimulation and organization of future behavior, making sense and meanings of current behavior, evaluation of life experience and current changes in person's life, in management of emotional processes. The construct of motivational potential of possible selves is developed, in which the notions of motivation and
\end{abstract}


self-concept are overlapped. The results of the studies are analyzed, which search for and determine parameters of possible selves that possess the most profound motivational potential. The study methods of possible selves are described, the most essential of which are questionnaires, interviews and experiments. In conclusion the unsolved issues of research in possible selves and perspectives for further study are outlined.

Keywords: possible selves, self-concept, motivating potential.

\section{References}

Abulkhanova-Slavskaya, K. A. (1991). Strategiya zhizni [Strategy of life]. Moscow: Mysl'.

Augustin of Hippo. (1998). Tvoreniya [Writings] (Vol. 1). Saint Petersburg/Kiev: Aleteiya/UTsIMMPress.

Belinskaya, E. P. (1999). Vremennye aspekty Ya-kontseptsii i identichnosti [Temporal aspects of selfconcept and identity]. Mir Psikhologii, 3, 140-147.

Bi, C., \& Oyserman, D. (2015). Left behind or moving forward? Effects of possible selves and strategies to attain them among rural Chinese children. Journal of Adolescence, 44, 245-258. doi:10.1016/ j.adolescence.2015.08.004

Bloom, K., Delmore-Ko, P., Masataka, N., \& Carli, L. (1999). Possible self as parent in Canadian, Italian, and Japanese young adults. Canadian Journal of Behavioural Science, 31(3), 198-207. doi:10.1037/h0087088

Bybee, J. A., \& Wells, Y. V. (2006). Body themes in descriptions of possible selves: Diverse perspectives across the life span. Journal of Adult Development, 13(2), 95-101. doi:10.1007/s10804-006-9009-9

Cameron, J. E. (1999). Social identity and the pursuit of possible selves: Implications for the psychological well-being of university students. Group Dynamics: Theory, Research, and Practice, 3(3), 179-189. doi:10.1037/1089-2699.3.3.179

Carver, C., Reynolds, S., \& Scheier, M. (1994). The possible selves of optimists and pessimists. Journal of Research in Personality, 28(2), 133-141. doi:10.1006/jrpe.1994.1011

Cross, S. E., \& Markus, H. R. (1994). Self-schemas, possible selves, and competent performance. Journal of Educational Psychology, 86(3), 423-438. doi:10.1037/0022-0663.86.3.423

Destin, M., \& Oyserman, D. (2010). Incentivizing education: Seeing schoolwork as an investment, not a chore. Journal of Experimental Social Psychology, 46(5), 846-849. doi:10.1016/j.jesp.2010.04.004

Dunkel, C. S. (2000). Possible selves as a mechanism for identity exploration. Journal of Adolescence, 23(5), 519-529. doi:10.1006/jado.2000.0340

Dunkel, C. S., \& Anthis, K. S. (2001). The role of possible selves in identity formation: a short-term longitudinal study. Journal of Adolescence, 24(6), 765-776. doi:10.1006/jado.2001.0433

Dunkel, C. S., Kelts, D., \& Coon, B. (2006). Possible selves as mechanisms of change in therapy. In C. Dunkel, J. \& Kerpelman (Eds.), Possible selves: Theory, research, and application (pp. 187-204). Huntington, NY: Nova.

Epshtein, M. N. (2001). Filosofiya vozmozhnogo [The philosophy of the possible]. Saint Petersburg: Aleteiya.

Erikson, M. (2007). The meaning of the future: Toward a more specific definition of possible selves. Review of General Psychology, 11(4), 348-358. doi:10.1037/1089-2680.11.4.348 
Frazier, L. D., Hooker, K., Johnson, P. M., \& Kaus, C. R. (2000). Continuity and change in possible selves in later life: A 5-year longitudinal study. Basic and Applied Social Psychology, 22(3), 237 243. doi:10.1207/S15324834BASP2203_10

Freeman, M., Hennessy, E., \& Marzullo, D. (2001). Defensive evaluation of antismoking messages among college-age smokers: The role of possible selves. Health Psychology, 20(6), 424-433. doi:10.1037//0278-6133.20.6.424

Freud, S. (2015). Ya i Ono [I and It]. Saint Petersburg: Azbuka. (Transl. of: Freud, S. (1923). Das Ich und das Es. Vienna: Internationaler Psychoanalytischer Verlag. (in German))

Hock, M. F., Deshler, D. D., \& Schumaker, J. B. (2006). Enhancing student motivation through the pursuit of possible selves. In C. Dunkel \& J. Kerpelman (Eds.), Possible selves: Theory, research, and application (pp. 205-221). Huntington, NY: Nova.

Hooker, K. (1992). Possible selves and perceived health in older adults and college students. Journal of Gerontology, 47(2), 85-95. doi:10.1093/geronj/47.2.P85

Hooker, K., \& Kaus, C. R. (1994). Health-related possible selves in young and middle adulthood. Psychology and Aging, 9(1), 126-133. doi:10.1037/0882-7974.9.1.126

Horney, K. (1997). Nevroz i lichnostnyi rost. Bor'ba za samoosushchestvlenie [Neurosis and personal growth. A fight for self-actualization]. Saint Petersburg: Vostochno-Evropeiskii Institut Psikhoanaliza i BSK. (Transl. of: Horney, K. (1950). Neurosis and human growth. New York: Norton).

Kao, G. (2000). Group images and possible selves among adolescents: Linking stereotypes to expectations by race and ethnicity. Sociological Forum, 15(3), 407-430. doi:10.1023/A:1007572209544

King, L. A., \& Hicks, J. A. (2007). Whatever happened to "What might have been?" Regrets, happiness, and maturity. American Psychologist, 62(7), 625-636. doi:10.1037/0003-066X.62.7.625

King, L. A., \& Patterson, C. (2001). Reconstructing life goals after the birth of a child with Down Syndrome: Finding happiness and growing. International Journal of Rehabilitation and Health, 5(1), 14-21. doi:10.1023/A:1012955018489

King, L. A., \& Raspin, C. (2004). Lost and found possible selves, subjective well-being, and Ego development in divorced women. Journal of Personality, 72(3), 603-632. doi:10.1111/j.00223506.2004.00274.x

King, L. A., \& Smith, N. G. (2004). Gay and straight possible selves: Goals, identity, subjective wellbeing, and personality development. Journal of Personality, 72(5), 967-994. doi:10.1111/j.00223506.2004.00287.x

Leontiev, D. A. (Ed.). (2011). Lichnostnyi potentsial: struktura i diagnostika [Personal potential: Structure and diagnostics]. Moscow: Smysl.

Landau, M. J., Keefer, L. A., Oyserman, D., \& Smith, G. C. (2014). The college journey and academic engagement: How metaphor use enhances identity-based motivation. Journal of Personality and Social Psychology, 106(5), 679-698. doi: 10.1037/a0036414

Markus, H., \& Nurius, P. (1986). Possible selves. American Psychologist, 41(9), 954-969. doi: 10.1037/0003-066X.41.9.954

Markus, H., \& Nurius, P. (1987). Possible selves: The interface between motivation and the self-concept. In K. Yardley \& T. Honess (Eds.), Self and identity: Psychosocial perspectives (pp. 157-172). Oxford: Wiley \& Sons.

Markus, H., \& Ruvolo, A. (2015). Possible selves: Personalized representations of goals. In L. A. Pervin (Ed.), Goal concepts in personality and social psychology (pp. 211-241). London/New York: Psychology Press. 
Morfei, M. Z., Hooker, K., Fiese, B. H., \& Cordeiro, A. M. (2001). Continuity and change in parenting possible selves: A longitudinal follow-up. Basic and Applied Social Psychology, 23(3), 217-223. doi:10.1207/153248301750433777

Oyserman, D., Bybee, D., \& Terry, K. (2006). Possible selves and academic outcomes: How and when possible selves impel action. Journal of Personality and Social Psychology, 91(1), 188-204. doi:10.1037/0022-3514.91.1.188

Oyserman, D., Bybee, D., Terry, K., \& Hart-Jonson, T. (2004). Possible selves as roadmaps. Journal of Research in Personality, 38(2), 130-149. doi:10.1016/S0092-6566(03)00057-6.

Oyserman, D., Destin, M., \& Novin, S. (2015). The context-sensitive future self: Possible selves motivate in context, not otherwise. Self and Identity, 14(2), 173-188. doi:10.1080/ 15298868.2014.965733

Oyserman, D., Gant, L., \& Ager, J. (1995). A socially contextualized model of African American identity: Possible selves and school persistence. Journal of Personality and Social Psychology, 69(6), 1216-1232. doi:10.1037/0022-3514.69.6.1216

Oyserman, D., \& Markus, H. (1990). Possible selves and delinquency. Journal of Personality and Social Psychology, 59(1), 112-125. doi:10.1037/0022-3514.59.1.112

Oyserman, D., Terry, K., \& Bybee, D. (2002). A possible selves intervention to enhance school involvement. Journal of Adolescence, 25(3), 313-326. doi:10.1006/jado.2002.0474

Petrovskiy, V. A. (2010). Chelovek nad situatsiei [Person above the situation]. Moscow: Smysl.

Petrovskiy, V. A. (2015). Subjectness as possibleness. Psychology. Journal of Higher School of Economics, 12(3), 86-130.

Rogers, C. (1997). Klientotsentrirovannaya psikhoterapiya [Client-centered psychotherapy]. Moscow/Kiev: Refl-buk/Vakler. (Transl. of: Rogers, C. (1951). Client-centered therapy: Its current practice, implications and theory. London: Constable).

Ryff, C. D. (1991). Possible selves in adulthood and old age: A tale of shifting horizons. Psychology and Aging, 6(2), 286-295. doi:10.1037/0882-7974.6.2.286

Starovoytenko, E. V. (2013). Capacities of the I in Relationship with the Other: Hermeneutics and Reflection. Psychology. Journal of Higher School of Economics, 10(4), 117-138. (in Russian)

Unemori, P., Omoregie, H., \& Markus, H. (2004). Self-portraits: Possible selves in EuropeanAmerican, Chilean, Japanese and Japanese-American cultural contexts. Self and Identity, 3(4), 321-338. doi:10.1080/13576500444000100

Whitty, M. (2002). Possible selves: Exploring the utility of a narrative approach. Identity: An International Journal of Theory and Research, 2(3), 213-230. doi:10.1207/S1532706XID0203_02

Ekaterina Yu. Vasilevskaya - postgraduate student, Faculty of Social Sciences, School of Psychology, Department of General and Experimental Psychology, National Research University Higher School of Economics.

Research area: self-concept, possible selves, motivation, psychology of personality.

E-mail: evasilevskaya@hse.ru

Olga N. Molchanova - professor, Faculty of Social Sciences, School of Psychology, Department of General and Experimental Psychology, National Research University Higher School of Economics, Ph.D., associate professor.

Research area: self-concept, self-esteem, developmental psychology.

E-mail: omolchanova@hse.ru 